\title{
BMJ Open Do we need to change catheter-related bloodstream infection surveillance in the Netherlands? A qualitative study among infection prevention professionals
}

\author{
Janneke DM Verberk (D) , ${ }^{1,2}$ Tjallie II van der Kooi, ${ }^{2}$ Lennie PG Derde, ${ }^{3}$ \\ Marc JM Bonten, ${ }^{4}$ Sabine C de Greeff, ${ }^{2}$ Maaike SM van Mourik ${ }^{1}$
}

To cite: Verberk JDM, van der Kooi TII, Derde LPG, et al. Do we need to change catheter-related bloodstream infection surveillance in the Netherlands? A qualitative study among infection prevention professionals. BMJ Open 2021;11:e046366. doi:10.1136/ bmjopen-2020-046366

- Prepublication history and additional supplemental material for this paper are available online. To view these files, please visit the journal online (http://dx.doi.org/10.1136/ bmjopen-2020-046366)

Received 27 October 2020 Accepted 04 August 2021

\section{Check for updates}

(C) Author(s) (or their employer(s)) 2021. Re-use permitted under CC BY-NC. No commercial re-use. See rights and permissions. Published by BMJ.

${ }^{1}$ Medical Microbiology and Infection Control, UMC Utrecht, Utrecht, The Netherlands ${ }^{2}$ Epidemiology and Surveillance, National Institute for Public Health and the Environment, Bilthoven, The Netherlands ${ }^{3}$ Department of Intensive Care Medicine, UMC Utrecht, Utrecht, The Netherlands

${ }^{4}$ Department of Medical Microbiology, UMC Utrecht, Utrecht, The Netherlands

Correspondence to Janneke DM Verberk; j.d.m.verberk-2@umcutrecht.nl

\section{ABSTRACT}

Objectives Catheter-related bloodstream infections (CRBSI) are a common healthcare-associated infection and therefore targeted by surveillance programmes in many countries. Concerns, however, have been voiced regarding the reliability and construct validity of CRBSI surveillance and the connection with the current diagnostic procedures. The aim of this study was to explore the experiences of infection control practitioners (ICPs) and medical professionals with the current CRBSI surveillance in the Netherlands and their suggestions for improvement. Design Qualitative study using focus group discussions (FGDs) with ICPs and medical professionals separately, followed by semistructured interviews to investigate whether the points raised in the FGDs were recognised and confirmed by the interviewees. Analyses were performed using thematic analyses.

Setting Basic, teaching and academic hospitals in the Netherlands.

Participants 24 ICPs and 9 medical professionals.

Results Main themes derived from experiences with current surveillance were (1) ICPs' doubt regarding the yield of surveillance given the low incidence of CRBSI, the high workload and IT problems; (2) the experienced lack of leadership and responsibility for recording information needed for surveillance and (3) difficulties with applying and interpreting the CRBSI definition. Suggestions were made to simplify the surveillance protocol, expand the follow-up and surveillance to homecare settings, simplify the definition and customise it for specific patient groups. Participants reported hoping for and counting on automatisation solutions to support future surveillance. Conclusions This study reveals several problems with the feasibility and acceptance of the current CRBSI surveillance and proposes several suggestions for improvement. This provides valuable input for future surveillance activities, thereby taking into account automation possibilities.

\section{INTRODUCTION}

Catheter-related bloodstream infections (CRBSIs) are common healthcare-associated infections (HAI). These infections are

\section{Strengths and limitations of this study}

- Focus group discussions and semistructured interviews allowed understanding and insights in opinions, ideas and feelings about the current method of surveillance of catheter-related bloodstream infections

- Infection control practitioners and medical professionals with a broad range of years of working experience from different types of hospitals participated in this study, representing almost a quarter of all Dutch hospitals.

- This study evaluated the surveillance of catheterrelated bloodstream infections in the Dutch setting. However, findings may be recognised by other countries that may experience similar problems with their surveillance and also looking for alternatives.

associated with prolonged hospital stay, increased risk of mortality and high costs, ${ }^{1-6}$ and therefore, one of the major infections targeted by surveillance programmes. ${ }^{7} 8$ Despite the substantial decline in CRBSI incidence achieved by implementation of preventive efforts, ${ }^{9-15}$ surveillance is still considered essential for identification of infections and for monitoring preventive interventions.

Many (national) surveillance programmes measure CRBSI rates systemically, however, all with slightly different definitions for CRBSI (box 1). ${ }^{16-18}$ The infection criteria used in the Dutch national CRBSI surveillance, coordinated by the surveillance network for HAI called PREZIES (Dutch acronym for 'PREventie van ZIEkenhuisinfecties door Surveillance'), come closest to those defined by the European Centre for Disease Prevention and Control (ECDC), although the PREZIES definition always requires a positive blood culture obtained via venepuncture to 
Box 1 Definitions of catheter-related bloodstream infection (CRBSI) and catheter-associated BSI

The National Healthcare Safety Network of the Centers for Disease Control and Prevention (CDC) provides the central line-associated BSI (CLABSI) defined as a primary laboratory-confirmed BSI where an eligible line is present. ${ }^{17}$

The European surveillance network healthcare-associated infections (HAl-NET), coordinated by the ECDC, applies a more strict definition relying on a laboratory-confirmed BSI in combination with either a catheter tip culture with the same microorganism or in combination with a positive culture with the same microorganism from pus from insertion site. Additionally, CRBSI can be scored by a quantitative blood culture ratio $>5$ of central venous catheter (CVC) blood and peripheral blood, or by differential period of 2 hours or more of positivity of blood cultures from peripheral blood and CVC blood (CRI-3-CVC). ${ }^{16}$ This CRBSI definition is a higher standard of proof of infection compared with the CDC's CLABSI definition.

For the Dutch surveillance, there are five definitions to score a CRBSI, all including clinical signs (fever, chills or hypotension) and absence of other focus of infection. The laboratory-confirmed CRBSI, which comes closest to the ECDC definition, requires also a combination of a positive tip culture along with a peripherally taken positive blood culture with the same microorganism. Next to this laboratory-confirmed definition, there are four other categories to define a CRBSI, trying to cover variations in local practices in the absence of culture results. An overview of the Dutch definitions is given in online supplemental appendix 1 .

meet criteria for a laboratory-confirmed CRBSI (online supplemental appendix 1). ${ }^{18}$ Box 2 summarises the Dutch CRBSI surveillance.

Within the Dutch surveillance network, yearly training meetings are organised for healthcare staff performing surveillance. During these meetings, concerns have been voiced regarding the reliability and construct validity of the CRBSI definition and the connection with the current diagnostic procedures. ${ }^{19-21}$ The possible misalignment between the CRBSI definition and diagnostic practices was confirmed by a Dutch study performed on an intensive care unit (ICU) in an academic hospital: they showed that only $2 \%$ of patients with a blood culture obtained for clinical reasons (ie, patients suspected of an infection) had appropriate microbiological diagnostics performed that would allow them to meet the surveillance requirements for a laboratory-confirmed CRBSI. This was mainly due to the lack of blood cultures obtained by venepuncture. ${ }^{22}$ Although never formally investigated, these experienced incompatibilities may be one of the reasons for the decreasing number of hospitals participating in the voluntary Dutch national surveillance over the past years. ${ }^{23}$ A 2016 surveillance protocol amendment mandating hospital-wide surveillance instead of ICU-only may also have contributed to the decline in participating centres.

These concerns with current CRBSI surveillance and the increasing availability of structured data stored in electronic health records (EHRs) creates a necessity to evaluate whether surveillance can be organised differently, preferably by incorporating automated options.
Box 2 The Dutch national surveillance strategy for catheter-related bloodstream infections (CRBSI)

In 2000, the Dutch National Surveillance Network PREventie van ZIEkenhuisinfecties door Surveillance (PREZIES) introduced a national CRBSI surveillance protocol which defines CRBSI and include instructions for the data collection procedure, aiming to align surveillance in hospitals. By executing the surveillance according to this protocol, hospitals can compare their results with aggregated national data, thereby helping starting interventions and improving patient care.

Hospitals include all central venous catheters and peripherally inserted catheters that are in situ for 48 hours in patients $\geq 18$ years. Tunnelled catheters are excluded from surveillance. Follow-up ends at day 28 , by catheter removal, mortality or by discharge at home or to another hospital. Hospitals are free to organise how they record the data, for example, manually entering data in digital files, or registering data into build-in surveillance packages within their electronic health record. After data collection is completed, hospitals can send their surveillance data to PREZIES by entering the data in an online registration system manually or uploading the data into this system directly according to standardised format. Hospitals can also send their data in a standardised data format to PREZIES.

Participation in all PREZIES surveillance programmes is voluntary, without any consequences related to performance, and hospitals pay a small fee to join the network. With a personal login they have access to an online reporting tool in which they can view their own data and their performance in relation to others (anonymously). Additionally, each year, PREZIES publishes an open, online report providing a summary of the national numbers and trends generated by the surveillance data.

Almost all studies evaluate surveillance programmes by investigating the effectiveness of a programme to reduce HAI. ${ }^{24-27}$ We feel that a more fundamental approach is needed to evaluate surveillance programmes and the quality of data it generates. For this purpose, we collected views and opinions of healthcare professionals involved in the surveillance that may help in designing future (automated) CRBSI surveillance. The aim of this study was (1) to explore the experiences of infection control practitioners (ICPs) and medical professionals with the current CRBSI surveillance in the Netherlands and (2) to collect their suggestions for an optimised CRBSI surveillance.

\section{MATERIALS AND METHODS}

From April to July 2019, face-to-face focus group discussions (FGDs) and semistructured interviews were conducted in order to gain understanding of professionals' experiences with the current CRBSI surveillance in the Netherlands and their suggestions for improvements. The supportive, non-judgemental setting offered by FGD enhances the likelihood of collecting diverse and spontaneous opinions, ideas and feelings. ${ }^{28}$ Semistructured interviews were used to validate the information collected in the FGDs.

\section{Participants}

In most hospitals, ICPs keep record of HAI, including CRBSI. When the hospital takes part in the national 
surveillance they perform the data collection according to the PREZIES protocol and annotate records according to the surveillance definition. As they are experienced with executing the surveillance, their opinion, experiences and views are important to investigate. ICPs from hospitals who indicated their intention to send in CRBSI surveillance data to PREZIES in 2017 or $2018(n=45)$ were invited to participate in an FGD by email via the PREZIESnetwork. FGDs were planned with all interested ICPs such that participants in each session represented different types of hospitals (academic, teaching and general hospitals) and concurrently allowed the largest number of ICPs to attend. ICPs who could not attend an FGD were asked to participate in an interview. Apart from ICPs, we included medical professionals in this study as they are often responsible for the surveillance of CRBSI, and its prevention in their patients. For the FGD with medical professionals, selection was performed using specificcriterion sampling: medical professionals were recruited by the research team via their professional network and chosen based on specific characteristics (medical specialty, gender, working in academic, teaching or general hospital, years of experience and experience in the field of infectious disease surveillance) ${ }^{29}$ Travel costs of all participants were reimbursed.

\section{Focus group discussions}

We organised two types of focus groups, involving ICPs and medical professionals separately. For the ICPs, consecutive FGDs were planned until no new themes were elicited, assessed by analysis alongside the FGDs. ${ }^{30}$ Thereafter, the FGD with medical professionals was planned. Each FGD was facilitated by the same moderator (JV) and observed by the same researcher (TvdK). We presented ourselves as researchers involved in the national CRBSI surveillance, and aimed to build rapport with the participants in order to encourage them to speak freely and to raise issues of importance to them. During each FGD, a topic guide was used (online supplemental appendix 2), however, participants were also allowed to go beyond the themes. The ICP FGDs were divided in two parts: first, the experiences with and opinions on the current CRBSI surveillance were discussed. Second, we asked for solutions for the problems that were raised in the first part of the FGD and for suggestions for a renewed surveillance regarding what to measure, how and in what patient groups. During the FGD with medical professionals more fundamental discussions were held about the entity of the infection, patient groups, benchmarking possibilities and suggestions for future surveillance activities (online supplemental appendix 2). Each FGD was held in Dutch, lasted approximately 2 hours and was audio recorded. Field notes were made during each FGD and incorporated in the analysis.

\section{Semistructured interviews}

The same topic list was used as for the ICPs' FGD, complemented with issues that emerged as important from the
FGDs. During the interviews, the interviewer investigated whether the points raised in the FGDs were recognised and confirmed by the interviewees.

\section{Data analysis}

The FGDs and interviews were audiorecorded and transcribed verbatim. The transcripts were used for thematic analysis performed by two researchers independently (JV and TvdK) using NVivo V.10 (QSR International, Melbourne, Australia). The first transcript of the first focus group was read closely and analysed by both researchers independently. The text units were inductively coded into categories and grouped in specific themes and subthemes, according to thematic analysis described by Braun and Clarke. ${ }^{31} 32$ We looked for themes which covered different socioecological aspects and the context (social, organisational and political factors). Both researchers discussed the data and correct interpretation, and an initial thematic map was developed. Transcripts from following FGDs were analysed and discrepancies were discussed. New themes were added to the map and if needed some previous transcripts were recoded. Themes were sorted and categorised in overarching themes resulting in a final thematic map. If controversy remained, another research team member (MvM) was consulted to aid decision making. The results of the ICP group were compared with the medical professionals' group to grasp diverse aspects of the participants' experiences and views. Besides, it was checked whether the themes derived from the FGDs were also covered in the interview transcripts. Findings are supported by a selection of quotes, translated to English for the purpose of this manuscript. As a quality check, these quotes were reverse-translated by another research team member $(\mathrm{SdG})$ to check their accuracy. The Consolidated criteria for Reporting Qualitative research guidelines were used for reporting the methods, analysis and results. ${ }^{33}$

\section{Patient and public involvement}

Patients and/or the public were not involved in the design, conduct, reporting or dissemination plans of this research.

\section{RESULTS}

\section{Response and participants}

Fourty-five hospitals had signed up in 2017 or 2018 to send in CRBSI surveillance data to PREZIES; of those, 23 hospitals $(51.1 \%)$ had (at least) one ICP that wanted to participate in this study. Eventually, 20 ICPs (from 19 hospitals) participated in an FGD. In total three FGDs were held with 6-7 participants each. After the FGDs, two interviews were conducted with four persons from two additional hospitals (duration $46.55 \mathrm{~min}$ and $43.15 \mathrm{~min}$, respectively) for the purpose of validating findings. This resulted in 24 participating ICPs (21 hospitals, response 46.6\%), representing approximately $25 \%$ of all Dutch hospitals. For the medical professionals, one FGD was held with nine 
Table 1 Characteristics of professionals participating in this study

\begin{tabular}{|c|c|}
\hline Characteristics & $\begin{array}{l}\text { Infection control practitioners } \\
(\mathrm{n}=24)\end{array}$ \\
\hline Gender; female/male & $23 / 1$ \\
\hline $\begin{array}{l}\text { Years of experience; mean } \\
\text { (range) }\end{array}$ & $12.1(2.5-29)$ \\
\hline \multirow{2}{*}{$\begin{array}{l}\text { Type of hospital*; general/ } \\
\text { teaching/academic (\% of } \\
\text { total Dutch hospitals in each } \\
\text { category) }\end{array}$} & $8 / 10 / 3(17.8 / 38.5 / 37.5)$ \\
\hline & Medical professionals $(n=9)$ \\
\hline Gender; female/male & $5 / 4$ \\
\hline $\begin{array}{l}\text { Years of experience; mean, } \\
\text { range }\end{array}$ & $10.1(1-20)$ \\
\hline Working areas & $\begin{array}{l}\text { Intensive care }(n=2) / \text { haematology } \\
(n=1) / \text { nephrology }(n=1) / \\
\text { paediatric infectious diseases } \\
(n=1) / \text { surgery }(n=1) / \text { nursing and } \\
\text { vascular access specialist }(n=1) / \\
\text { medical microbiology }(n=1) / \\
\text { research }(n=1)\end{array}$ \\
\hline
\end{tabular}

${ }^{*}$ Corrected for hospital level as for one hospital two infection control practitioners participated in focus group discussions and interviews were held with two infection control practitioners per hospital.

medical professionals (doctors, nurse, researcher) from seven specialties and from four different centres. The characteristics of the professionals that participated in an FGD or interview are presented in table 1. All medical professionals were aware of the existence of the national CRBSI surveillance and six were actively involved in the CRBSI surveillance of their hospital.

\section{Experiences with the current CRBSI surveillance}

Thematic analysis identified three main themes with nine subthemes from the experiences of the participants with the current CRBSI surveillance (table 2).

\section{Theme 1: balancing the costs and benefits of performing surveillance}

One main theme-with four subthemes-that arose from the data was the balance between the effort that was put into surveillance activities compared with the benefits.

\section{CRBSI surveillance is valuable to monitor quality of care}

The main reason for all participants to perform surveillance was to generate insights in their CRBSI incidence. According to ICPs, the surveillance results led to opportunities for quality improvement, raised awareness for infection prevention (eg, in management layers) and initiated conversations about current practices for example, one ICP gave the example that a peak in the CRBSI surveillance data initiated an assessment about the method of catheter insertion and method of care and how they could be improved.
Table 2 Overview of themes and subthemes

\begin{tabular}{ll}
\hline Main theme & Subtheme \\
\hline $\begin{array}{l}\text { Current surveillance } \\
\text { benefits }\end{array}$ & $\begin{array}{l}\text { CRBSI surveillance is valuable to } \\
\text { monitor quality of care } \\
\text { Low incidence } \\
\text { IT problems } \\
\text { Performing surveillance is too } \\
\text { labour-intensive }\end{array}$ \\
$\begin{array}{ll}\text { 2. Lack of leadership and } \\
\text { responsibility }\end{array}$ & $\begin{array}{l}\text { Criteria do not cover all CRBSIs } \\
\text { Criteria not applicable to all } \\
\text { definition }\end{array}$ \\
& $\begin{array}{l}\text { patient groups } \\
\text { Criteria not in line with } \\
\text { diagnostic practice }\end{array}$ \\
& $\begin{array}{l}\text { Large variability in interpretation } \\
\text { Difficulties in causally relating } \\
\text { symptoms to central line }\end{array}$
\end{tabular}

\begin{tabular}{|c|c|}
\hline \multicolumn{2}{|c|}{ Ideas and suggestions for improvement } \\
\hline A. Simplified surveillance & $\begin{array}{l}\text { Simpler inclusion criteria and } \\
\text { endpoints } \\
\text { Longer follow-up }\end{array}$ \\
\hline \multicolumn{2}{|l|}{$\begin{array}{l}\text { B. Extension surveillance to } \\
\text { homecare setting }\end{array}$} \\
\hline $\begin{array}{l}\text { C. Modification of the } \\
\text { definition }\end{array}$ & $\begin{array}{l}\text { Definition customised for } \\
\text { specific patient groups } \\
\text { No CBRSI subcategories }\end{array}$ \\
\hline \multicolumn{2}{|c|}{ D. More automated solutions } \\
\hline $\begin{array}{l}\text { E. Alternative surveillance } \\
\text { options }\end{array}$ & $\begin{array}{l}\text { Surveillance of tip colonisation } \\
\text { Shorten the period of executing } \\
\text { surveillance } \\
\text { Surveillance of other catheter } \\
\text { types }\end{array}$ \\
\hline
\end{tabular}

CRBSI, catheter-related bloodstream infection; IT, information technology.

For us it is mainly a quality indicator, we just want to keep numbers as low as possible and the only way to find that out is to register infections. (FGD-MP05)

\section{Low incidence}

Some ICPs were doubtful whether it is worthwhile continuing the CRBSI surveillance, given their stable low incidence over the years and the many hours they spend on executing surveillance.

\section{Information technology problems}

Most hospitals use a module or package in their EHR to collect and record surveillance data: the stage of development and functionalities vary by hospitals. However, there is a broad variety in information technology (IT) problems infection prevention departments face and the IT support they receive: there are experiences with uncooperative EHR-developers and technical problems 
of systems, problems with extracting the data from the system, and the feeling that it is very difficult to get things done in this field.

I find that in practice, it is very difficult to get the automation department to do things anyway. I think that's the experience in all hospitals. (FGD-ICP017)

ICPs feel they often have to arrange and co-develop EHR improvements, however, according to them it is not their responsibility or skill.

Look, of course we want to know the numbers, but as long as the automation department is holding us back...WE are not going to do this as an infection prevention department. That is not possible, we don't want to, and we don't have the time to do it! (FGD-ICP03)

Last, for many health professionals it is unclear what the automatisation possibilities are. They admit that they don't have the knowledge or interest; nevertheless, they do have high expectations of it.

\section{Performing surveillance is too labour-intensive}

Performing surveillance is too labour-intensive and timeintensive according to all participants. One ICP called it demotivating. Additionally, the high work pressure people already experience in general and the recent extension to CRBSI hospital-wide surveillance were mentioned. Executing and coordinating surveillance takes too much time at the expense of other infection prevention tasks. For this reason, many hospitals have data managers, students or administrative support helping with performing the surveillance.

But yes, it is very a time investment and, so to say, the input does not completely weigh up to the benefit. (FGD-ICP09)

Because people are overloaded, they have so much to do already! (FGD-ICP06)

The main reason for the high workload is missing data in the EHR. In most hospitals, healthcare professionals register information in the EHR that ICPs use for the surveillance: after catheter insertion the catheter is registered into the system, nurses add (daily) information about the use of and possible complications associated with the catheter and record catheter removal. In some hospitals the information entered in the system is automatically extracted and ICPs complement some data fields required for surveillance. In other cases ICPs fill in surveillance data based on free-text notes. ICPs unanimously agreed that information, such as the origin of blood samples, is often missing or not filled in properly. In particular the removal date of the catheter is often lacking, thereby complicating the calculation of line days needed for the denominator of reporting the incidence. Looking up the removal date in free-text clinical notes is experienced as burdensome.
If they don't enter the removal date [of the catheter] you are searching for hours to find it. (FGD-ICP02)

This incorrect and missing data lead to incorrect surveillance results. ICPs repeatedly reported that they are aware of incomplete or incorrect surveillance data, however, they feel they cannot help it.

We run into a lot of issues and actually, every now and then it seems we generate numbers that are based on shots in the dark.... (FGD-ICP04)

\section{Theme 2: lack of leadership and responsibility: ICPs filling the gap}

The ICPs as well as the medical professionals agreed that the responsibility for the treatment of the patient and the handling of the catheter lies with the treating physician, and both the medical specialists and nurses are responsible for the care. However, as described above, the information needed to perform CRBSI surveillance is often not fully available from the EHRs. According to the ICPs this is because the people responsible for the patient lack involvement with and interest for surveillance and, hence, responsibility for registering information. Although responsibilities and actions are often described in protocols and documents, ICPs emphasise that practice is not always in accordance with hospital-wide agreements.

In principle, everything you have to do is described, but then... you still can do something different. (FGD-ICP17)

I can't get them to do it! Manually entering data in the system when ordering a blood-or tip culture. They just don't do it. (FGD-ICP02)

Most ICPs stated that in the above-mentioned situations they take the responsibility to complete the surveillance records by looking up or asking for data, but they feel that it is not their responsibility to do so: they should have a coordinating role and are responsible for the CRBSI reports. Medical professionals agreed and confirmed that information is lacking in the EHR, however, they did not provide any suggestions to improve this or who's responsibility it is.

\section{Theme 3: problems with the CRBSI definition}

\section{Criteria do not cover all CRBSIs}

ICPs and medical professionals reported several problems regarding the definition that is used in surveillance. First, they emphasised that there are some cases that cannot be scored as a CRBSI according to surveillance criteria, but that are seen as CRBSI according to ICPs or medical doctors. Therefore, they feel that the surveillance does not catch all CRBSIs.

There are CRBSIs that fall outside the criteria of PREZIES. And those, yes those, are the ones you are interested in. (FGD-ICP07) 
Criteria are not applicable to all patient groups

In the current surveillance, the same definitions are used for all patient groups with a central venous catheter (CVC) or peripherally inserted central catheter (PICC). According to both ICPs and medical professionals, these definitions are not applicable to all patient groups, in particular haematology patients as their clinical symptoms caused by neutropenia are indistinguishable from infection symptoms.

\section{Criteria are not in line with diagnostic practice}

Clinical practice is often not in line with the strict criteria to define CRBSIs, making them difficult to score: for example, the blood and tip cultures are not taken within 24 hours of each other, blood samples are obtained via the CVC instead of through a venepuncture, or antibiotics are started before cultures are obtained.

Every now and then you just know for sure that it is one [a CRBSI], but then, for example, the blood culture was taken two hours too late to meet the definition. Very frustrating. (Int-ICP22)

Medical doctors confirmed and emphasised that the current surveillance protocol is not in line with their clinical practice.

\section{Large variability in interpretation}

Some ICPs experience a large variability in interpretation of the definition and they therefore question the potential for benchmarking.

\section{Difficulties in causally relating symptoms to central line}

Last, all participants concluded that patients with a CVC or PICC suspected for infection are complex, and the causes of certain clinical symptoms are not always clear. Causally relating the symptoms to the CVC is therefore difficult. They stated that a CRBSI is sometimes used as a 'residual diagnosis', if no other focus or explanation can be found.

\section{Suggestions for improvement}

Five main themes for improvement were addressed: (A) a simplified surveillance; (B) extending the surveillance to homecare settings; (C) modification of the definition; (D) more automated surveillance and (E) suggestions for alternative surveillance options (table 2). Below a short description is given per theme.

\section{Theme A: wish for a simplified surveillance}

The wish for a more simplified surveillance methodology recurred throughout the dataset. The current surveillance is experienced as too complicated regarding the inclusion criteria for catheters, the information nurses have to enter into EHR systems and the exact endpoints of the surveillance. ICPs brought forward the possibility of registering the incidence only, without collecting additional information for the purpose of casemix correction, thereby accepting the possible bias. The medical professionals disagreed: they felt casemix correction is essential to have reliable benchmarking. Both groups agreed that the follow-up was considered too short and should be longer than 28 days.

\section{Theme B: extending surveillance to homecare setting}

Many participants also agreed on the need to extend the current surveillance protocol to patients in homecare settings. Both ICPs and medical professionals argued that they are still responsible for the patient when discharged with a CVC or PICC in situ. Given the increasing trend of shared healthcare and short hospital stays it is valuable to prolong the follow-up for patients who are discharged with a PICC and regularly return to the hospital for check-up visits.

"[....] while more and more patients are treated outside the hospital, and these are completely excluded form surveillance. And in my opinion, that's a shortcoming if you see that length of hospitals stays are becoming shorter and patients are treated under responsibility of the hospital, and we don't pay attention to that." (FGD-MP07)

\section{Theme C: modification of the definition}

The definition used in surveillance was a recurrent theme in all FGDs and interviews. The wish for an adaptation of this definition was even communicated as a compelling advice rather than a suggestion. Especially the medical professionals advised to adopt a more tailored surveillance definition for specific target groups as the current definitions are not applicable to groups such as haematology patients or children (though the latter is currently not included in national surveillance). ICPs agreed on this and also suggested to create one single definition per patient group instead of the current four categories to score a CRBSI (see box 1 ).

\section{Theme D: more automated solutions}

According to all participants much more could be developed, improved and achieved by local automation processes. Their expectations of IT possibilities are high and seen as the solution for workload reduction as it can help with easily recording data, and for the problems they face regarding definition subjectivity through the use of advanced algorithms. Second, ICPs would appreciate if changes to the national surveillance protocol are aligned between the national surveillance organisation PREZIES and EHR-software companies. ICPs often have to arrange changes in EHR systems while they feel it is not their responsibility and they are not comfortable with it.

"Yes and maybe it is very idealistic, but it would be very appreciated if...when for example PREZIES is going to change something in the CRBSI surveillance that there is contact with the most common EHR suppliers, and that PREZIES is not saying to hospitals every time: 'it is your problem to fix it in your EHR. Go and find out yourself how you will implement it.' (FGD-ICP02) 
Theme E: suggestions for alternative surveillance options

It was argued that catheter tip colonisation combined with information about the causative microorganism would give more steering information as it is a harbinger of infection and occurs more frequent than the strict definition requiring a positive blood and tip culture. Other suggestion was to reduce the current registration period, for example a registration of 3 months per year, in order to reduce the workload.

We just take one month and then we add additional information to see what is happening over there. You then measure a sort of prevalence. Because now you...you... you are looking for all those catheters the entire year...well you become completely crazy! (Int-ICP24)

ICPs indicated they would like to have the possibility to (temporary) monitor other types of catheters, for example, arterial lines, or specific patient groups or wards. This was contradicted by the medical professionals who argued to stick to CVCs and PICCs for national surveillance for benchmarking purposes, however, to include tunnelled central lines.

None of the participants was interested in shifting the focus of national surveillance from CRBSI to other endpoints such as hospital-acquired bloodstream infections (BSIs) or to measure other quality indicators instead of CRBSI, such as ventilator-associated pneumonia for benchmarking purposes.

\section{DISCUSSION}

This study revealed multiple problematic factors that ICPs experience in performing the current CRBSI surveillance. Surveillance is experienced as too labourintensive and complicated with respect to applying the definition, the lack of responsibility in recording data and IT-related problems. To optimise national surveillance, professionals agreed to adapt the surveillance definition to specific patient groups and extending the duration of follow-up and the surveillance to home care. It became clear that all professionals look forward to automation options to facilitate data collection and improve standardisation of surveillance. There were different opinions about the types of catheters to include in surveillance: medical professionals suggested to continue CVC and PICC surveillance, however, including tunnelled catheters, while ICPs were more in favour of more choice in selecting the type of catheter under surveillance and were less interested in benchmarking.

A comparable study was performed in Sweden by Ridelberg and Nilsen ${ }^{34}$ : by interviewing 22 ICPs they evaluated obstacles concerning the surveillance process of their biannual point prevalence surveys, focusing on the challenges in using HAI results. Although this study evaluated a different type of surveillance, similar obstacles were reported such as limited involvement of clinical staff and nurses and shortage of personnel resources and technical problems. The high workload and labour-intensiveness is mentioned by several other surveys and quantitative studies and is therefore a recurrent theme in performing surveillance. ${ }^{35-37}$

Almost all studies evaluating surveillance programmes focus mainly on the effectiveness to reduce HAI. ${ }^{24-27}$ We feel that a more fundamental approach is needed to evaluate surveillance programmes and the quality of data it generates. In our opinion, evaluating surveillance should also include a discussion about the effort taken to collect these data, what we actually measure and how. Collecting meanings and views about current surveillance activities in a systematic way is valuable as it enables the discovery of barriers that should be tackled to make surveillance more relevant, effective, feasible and reliable. The value of using CRBSI for benchmarking has been questioned due to high variability in CRBSI rates caused by differences in diagnostic practices, subjective interpretation, and CRBSI rates close to zero limiting meaningful comparisons. ${ }^{21} 26{ }^{38-42}$ Hence, effort is put into development of alternative definitions of CRBSI in different patient groups $^{43-45}$ or for alternative quality outcome measures for benchmarking such as hospital-onset bacteraemia. ${ }^{46} 47$

The development of automated surveillance methods is another strategy to improve the quality of CRBSI surveillance, which limits subjectivity and reduces the time investment. Several research groups developed automated algorithms to detect BSIs in patients with a catheter. ${ }^{48-51}$ Despite good performances, all used the CDC definition for automation (central line-associated BSI). To the best of our knowledge, no examples are available for automation using the CRBSI definition, which is a higher standard of proof that the infection is related to the CVC. Additionally, automation does not change the social practices and obstacles of the data collection as described by Dixon-Woods et al. ${ }^{42}$ This study, therefore, first identified views and opinions about the current surveillance to guide further development of CRBSI surveillance and automation solutions in the future. Designing IT solutions without identifying underlying problems and views may fail to be helpful and to be accepted in the field. The in-depth information generated in this study is also meant as a preparation for a planned future quantitative study on (semi) automatisation of CRBSI surveillance.

\section{Strengths and limitations}

We collected opinions from professionals from different types of hospitals with a broad range of years of working experience. We included ICPs as the surveillance coordinators using the data, but also medical professionals: the latter have a different view on benchmarking and national surveillance purposes compared with the ICPs. Unfortunately, only one nurse, specialised in vascular access devices, could attend the FGD.

Views from general hospitals may be slightly underrepresented in this study, as only about $18 \%$ of all Dutch general hospitals participated in our FGDs. These hospitals may experience less difficulties in organising and 
conducting surveillance, however, as brought forward during the FGDs, this is because data collection is performed mainly manually. During the FGDs it became clear that ICPs from small hospitals share the desire to have more IT solutions, but lack knowledge and expertise in this field. Second, it is possible that hospitals that do not encounter problems are less interested to participate in these FGDs. However, the unexpected high response rate $(51.1 \%)$-representing a quarter of all Dutch hospitals-could be considered indicative of the problems in current surveillance activities. Alternatively, non-response may be related to the current shortage of ICP personnel in Dutch hospitals; therefore, not having time to travel and participate in the FGDs. It would be interesting to hear from ICPs of hospitals that do not participate in national surveillance about their reasons for non-participation. However, because we were interested in the experiences with performing the current surveillance we only recruited ICPs via the PREZIES network. Last, this study is performed in a Dutch setting and evaluated the Dutch national CRBSI surveillance. Nevertheless, findings may be recognised by other countries, who may experience similar problems and are also looking for surveillance alternatives.

\section{CONCLUSION}

This study describes shortcomings in the current Dutch CRBSI surveillance. According to the participants of this study, the surveillance is considered too labour-intensive, restricted by IT-related problems and some perceived that the benefits of surveillance do not outweigh the time-investment required from the ICPs, given the low CRBSI incidence. Moreover, the CRBSI-definition is not aligned with daily diagnostic practice and there is a lack of responsibility in recording data required for surveillance. Suggested improvements include: a modification of the definition customised to specific patient groups, automatisation options to improve data collection, standardisation and to reduce workload, and to include catheters in homecare situations and extend the follow-up period. These results provide valuable input for making decisions for future surveillance activities, taking into account automation possibilities.

\section{Twitter Maaike SM van Mourik @vanmourikmaaike}

Acknowledgements We gratefully acknowledge the time and enthusiasm of the professionals who participated in the focus groups or interviews. We would like to thank R. Raymakers and T. van der Bruggen for clinical advice regarding this study and S. Tonkin-Crine for advice regarding writing this manuscript.

Contributors JV was responsible for the study conception, design, data collection, analyses and interpretation, as well as for drafting the manuscript, revision and final approval. TvdK was involved in the data collection and the data analysis. MvM and LD supervised the study. TvdK, SdG, LD, MvM and MB made critical revisions to the paper. All authors approved the final manuscript.

Funding The authors have not declared a specific grant for this research from any funding agency in the public, commercial or not-for-profit sectors.

Competing interests None declared.

Patient consent for publication Not required.
Ethics approval This study was reviewed by the Medical Institutional Review Board of the University Medical Centre Utrecht and was considered not to fall under the Medical Research Involving Human Subjects Act (ref. no. 19/161). Written informed consent was obtained from all participants of this study.

Provenance and peer review Not commissioned; externally peer reviewed. Data availability statement № data are available.

Supplemental material This content has been supplied by the author(s). It has not been vetted by BMJ Publishing Group Limited (BMJ) and may not have been peer-reviewed. Any opinions or recommendations discussed are solely those of the author(s) and are not endorsed by BMJ. BMJ disclaims all liability and responsibility arising from any reliance placed on the content. Where the content includes any translated material, BMJ does not warrant the accuracy and reliability of the translations (including but not limited to local regulations, clinical guidelines, terminology, drug names and drug dosages), and is not responsible for any error and/or omissions arising from translation and adaptation or otherwise.

Open access This is an open access article distributed in accordance with the Creative Commons Attribution Non Commercial (CC BY-NC 4.0) license, which permits others to distribute, remix, adapt, build upon this work non-commercially, and license their derivative works on different terms, provided the original work is properly cited, appropriate credit is given, any changes made indicated, and the use is non-commercial. See: http://creativecommons.org/licenses/by-nc/4.0/.

\section{ORCID iD}

Janneke DM Verberk http://orcid.org/0000-0003-2148-5935

\section{REFERENCES}

1 Tacconelli E, Smith G, Hieke K, et al. Epidemiology, medical outcomes and costs of catheter-related bloodstream infections in intensive care units of four European countries: literature- and registry-based estimates. J Hosp Infect 2009;72:97-103.

2 Blot SI, Depuydt P, Annemans L, et al. Clinical and economic outcomes in critically ill patients with nosocomial catheter-related bloodstream infections. Clin Infect Dis 2005;41:1591-8.

3 Stevens V, Geiger K, Concannon C, et al. Inpatient costs, mortality and 30-day re-admission in patients with central-line-associated bloodstream infections. Clin Microbiol Infect 2014;20:0318-24.

4 Fletcher S. Catheter-Related bloodstream infection. Cont Educ Anaesthesia Crit Care Pain 2005;5:49-51.

5 Walker G, Todd A. Nurse-Led PICC insertion: is it cost effective? Br J Nurs 2013;22:S9-15.

6 Ziegler MJ, Pellegrini DC, Safdar N. Attributable mortality of central line associated bloodstream infection: systematic review and metaanalysis. Infection 2015;43:29-36.

7 Magill SS, Edwards JR, Bamberg W, et al. Multistate pointprevalence survey of health care-associated infections. N Engl J Med 2014;370:1198-208.

8 Haque M, Sartelli M, McKimm J, et al. Health care-associated infections - an overview. Infect Drug Resist 2018;11:2321-33.

9 Umscheid CA, Mitchell MD, Doshi JA, et al. Estimating the proportion of healthcare-associated infections that are reasonably preventable and the related mortality and costs. Infect Control Hosp Epidemiol 2011;32:101-14.

10 Berenholtz SM, Lubomski LH, Weeks K, et al. Eliminating central line-associated bloodstream infections: a national patient safety imperative. Infect Control Hosp Epidemiol 2014;35:56-62.

11 Pronovost $\mathrm{P}$, Needham D, Berenholtz S, et al. An intervention to decrease catheter-related bloodstream infections in the ICU. N Engl J Med 2006;355:2725-32.

12 Centers for Disease Control and Prevention (CDC). Vital signs: central line-associated blood stream infections-United States, 2001, 2008, and 2009. MMWR Morb Mortal Wkly Rep 2011;60:243-8.

13 Hallam C, Jackson T, Rajgopal A, et al. Establishing catheter-related bloodstream infection surveillance to drive improvement. J Infect Prev 2018;19:160-6.

14 Bion J, Richardson A, Hibbert P, et al. 'Matching Michigan': a 2-year stepped interventional programme to minimise central venous catheter-blood stream infections in intensive care units in England. BMJ Qual Saf 2013;22:110-23.

15 Almirante B, Limón E, Freixas N, et al. Laboratory-Based surveillance of hospital-acquired catheter-related bloodstream infections in Catalonia. Results of the VINCat program (2007-2010). Enferm Infecc Microbiol Clin 2012;30(Suppl 3):13-19.

16 European Centre for Disease Prevention and Control. HealthcareAssociated infections acquired in intensive care units. contract No.: 
annual epidemiological report for 2015. Stockholm European Centre for Disease Prevention and Control; 2017.

17 National Healthcare Safety Network (NHSN). Patient safety component manual. Atlanta CDC; 2019.

18 PREZIES. Protocol en dataspecificaties, module Lijnsepsis Bilthoven: National Institute for public health and the environment, 2020. Available: https://www.rivm.nl/sites/default/files/2019-09/lijnsepsis\% 20protocol\%202020\%20versie\%201.01.pdf [Accessed 18 Mar 2020].

19 Timsit J-F, Rupp M, Bouza E, et al. A state of the art review on optimal practices to prevent, recognize, and manage complications associated with intravascular devices in the critically ill. Intensive Care Med 2018;44:742-59.

20 Worth LJ, Brett J, Bull AL, et al. Impact of revising the National nosocomial infection surveillance system definition for catheterrelated bloodstream infection in ICU: reproducibility of the National healthcare safety network case definition in an Australian cohort of infection control professionals. Am J Infect Control 2009;37:643-8.

21 Cherifi S, Mascart G, Dediste A, et al. Variations in catheter-related bloodstream infections rates based on local practices. Antimicrob Resist Infect Control 2013;2:10.

22 Sips ME, Bonten MJM, Van Mourik MSM. Reliability and feasibility of surveillance of central line-associated bloodstream infections in an ICU setting: ECCMID 2017 Vienna, Austria, 2017. Available: https://iris.unipa.it/retrieve/handle/10447/237123/440424/ECCMID_ FinalProgramme Vienna Web.pdf [Accessed 21 Apr 2020].

23 PREZIES. Referentiecijfers 2014-2018: Lijnsepsis Bilthoven: National Institute for public health and the environment, 2019. Available: https://www.rivm.nl/sites/default/files/2019-09/Referentiecijfers\% 202019\%20versie\%201.0.pdf [Accessed 18 Mar 2020].

24 Wilson JA, Ward VP, Coello R, et al. A user evaluation of the nosocomial infection national surveillance system: surgical site infection module. J Hosp Infect 2002;52:114-21.

25 Hajdu A, Eriksen HM, Sorknes NK, et al. Evaluation of the National surveillance system for point-prevalence of healthcare-associated infections in hospitals and in long-term care facilities for elderly in Norway, 2002-2008. BMC Public Health 2011;11:923.

26 Niedner MF, 2008 National Association of Children's Hospitals and Related Institutions Pediatric Intensive Care Unit Patient Care FOCUS Group. The harder you look, the more you find: catheterassociated bloodstream infection surveillance variability. Am J Infect Control 2010;38:585-95.

27 Gastmeier P, Sohr D, Schwab F, et al. Ten years of KISS: the most important requirements for success. J Hosp Infect 2008;70 Suppl 1:11-16.

28 Powell RA, Single HM. Focus groups. Int J Qual Health Care 1996;8:499-504.

29 Palys T. Purposive sampling. In: The SAGE encyclopedia of qualitative research methods. Los Angeles: Sage, 2008: 2. 697-8.

30 Braun V, Clarke V. To saturate or not to saturate? Questioning data saturation as a useful concept for thematic analysis and sample-size rationales. Qual Res Sport Exerc Health 2021;13:201-16.

31 Braun V, Clarke V. Using thematic analysis in psychology. Qual Res Psychol 2006;3:77-101.

32 Braun V, Clarke V, Hayfield N. Thematic Analysis. In: Liamputtong P, ed. Handbook of research methods in health social sciences. Singapore: Springer, 2019: 843-60.

33 Tong A, Sainsbury P, Craig J. Consolidated criteria for reporting qualitative research (COREQ): a 32-item checklist for interviews and focus groups. Int J Qual Health Care 2007;19:349-57.
34 Ridelberg M, Nilsen P. Using surveillance data to reduce healthcareassociated infection: a qualitative study in Sweden. J Infect Prev 2015;16:208-14.

35 Mitchell BG, Hall L, Halton K, et al. Time spent by infection control professionals undertaking healthcare associated infection surveillance: a multi-centred cross sectional study. Infect Dis Health 2016:21:36-40.

36 Hall L, Halton K, Macbeth D, et al. Roles, responsibilities and scope of practice: describing the 'state of play' for infection control professionals in Australia and New Zealand. Healthc Infect 2015;20:29-35.

37 Grota PG, Stone PW, Jordan S, et al. Electronic surveillance systems in infection prevention: organizational support, program characteristics, and user satisfaction. Am J Infect Control 2010;38:509-14.

38 Kourkouni E, Kourlaba G, Chorianopoulou E, et al. Surveillance for central-line-associated bloodstream infections: accuracy of different sampling strategies. Infect Control Hosp Epidemiol 2018;39:1210-5.

39 Larsen EN, Gavin N, Marsh N, et al. A systematic review of centralline-associated bloodstream infection (CLABSI) diagnostic reliability and error. Infect Control Hosp Epidemiol 2019;40:1100-6.

$40 \mathrm{Limb}$ M. Variations in collecting data on central line infections make comparison of hospitals impossible, say researchers. BMJ 2012;345:e6377.

41 Mermel LA. Defining intravascular catheter-related infections: a plea for uniformity. Nutrition 1997;13:2s-4.

42 Dixon-Woods M, Leslie M, Bion J, et al. What counts? An ethnographic study of infection data reported to a patient safety program. Milbank Q 2012;90:548-91.

43 de Grooth HJ, Timsit J-F, Mermel L, et al. Validity of surrogate endpoints assessing central venous catheter-related infection: evidence from individual- and study-level analyses. Clin Microbiol Infect 2020;26:563-571.

44 Rijnders BJA, Van Wijngaerden E, Peetermans WE. Catheter-tip colonization as a surrogate end point in clinical studies on catheterrelated bloodstream infection: how strong is the evidence? Clin Infect Dis 2002;35:1053-8.

45 Franklin JA, Gaur AH, Shenep JL, et al. In situ diagnosis of central venous catheter-related bloodstream infection without peripheral blood culture. Pediatr Infect Dis J 2004;23:614-8.

46 Rock C, Thom KA, Harris AD, et al. A multicenter longitudinal study of Hospital-Onset bacteremia: time for a new quality outcome measure? Infect Control Hosp Epidemiol 2016;37:143-8.

47 Leekha S, Li S, Thom KA, et al. Comparison of total hospitalacquired bloodstream infections to central line-associated bloodstream infections and implications for outcome measures in infection control. Infect Control Hosp Epidemiol 2013;34:984-6.

48 Trick WE, Zagorski BM, Tokars Jl, et al. Computer algorithms to detect bloodstream infections. Emerg Infect Dis 2004;10:1612-20.

49 Lin MY, Woeltje KF, Khan YM, et al. Multicenter evaluation of computer automated versus traditional surveillance of hospitalacquired bloodstream infections. Infect Control Hosp Epidemiol 2014:35:1483-90.

50 Woeltje KF, Butler AM, Goris AJ, et al. Automated surveillance for central line-associated bloodstream infection in intensive care units. Infect Control Hosp Epidemiol 2008;29:842-6.

51 Hota B, Lin M, Doherty JA, et al. Formulation of a model for automating infection surveillance: algorithmic detection of centralline associated bloodstream infection. J Am Med Inform Assoc 2010;17:42-8. 